\title{
Hyperkalemia developed from atorvastatin after kidney transplantation: a case report
}

\author{
Tae Hyun Ban ${ }^{1}$, Bum Soon Choi ${ }^{1}$, Mi-Hyeong Kim², Jeong-Kye Hwang ${ }^{2}$, Jihyang Lim ${ }^{3}$ \\ ${ }^{1}$ Division of Nephrology, Department of Internal Medicine, The Catholic University of Korea, Eunpyeong St. Mary's Hospital, Seoul, Korea \\ ${ }^{2}$ Division of Transplantation Surgery, Department of Surgery, The Catholic University of Korea, Eunpyeong St. Mary's Hospital, Seoul, Korea \\ ${ }^{3}$ Department of Laboratory Medicine, The Catholic University of Korea, Eunpyeong St. Mary's Hospital, Seoul, Korea
}

Background: Hyperkalemia early after kidney transplantation (KT) is one of common complications owing to immunosuppressive agents, prophylactic antibiotics, and uncontrolled diabetes. However, it is difficult to predict that uncommon origins are the primary cause of hyperkalemia. Here we report a case of hyperkalemia in a KT recipient after administering atorvastatin.

Methods: A 64-year-old male patient, who underwent hemodialysis for 54 months due to end-stage kidney disease by type 2 diabetes, received deceased donor KT. After transplantation, he was considering discharge without any complications. But his laboratory results showed severe hyperkalemia right before discharge.

Results: The patient showed hypokalemia for 1 week after $\mathrm{KT}$, but his laboratory results revealed hyperkalemia of $6.4 \mathrm{mmol} / \mathrm{L}$ on the 12th day. Therefore, he was treated four times a day with Kayexalte enema and oral Kayexalte supplementation. However, serum potassium level increased to $7.3 \mathrm{mmol} / \mathrm{L}$ on the 13th day. Until that time, serum tacrolimus level was continuously maintained between 5 and $9 \mathrm{ng} / \mathrm{mL}$ under trimethoprim-sulfamethoxazole administration and proper control of diabetes via basal insulin. Serum renin, aldosterone, and aldosterone-to-renin ratio were normal. There was an addition of atorvastatin 4 days before the onset of hyperkalemia. After review of rationale, atorvastatin was discontinued due to the possibility of causative agent of hyperkalemia. The transtubular potassium gradient (TTKG) decreased from 7 for the 1 st day and 5 for the 5th day to 1 on the 13th day after transplantation. Serum potassium level of the patient was maintained at $5 \mathrm{mmol} / \mathrm{L}$ under potassium lowering agent on the 16th day. Three weeks after transplantation, the TTKG was 2 and serum potassium level was $5.4 \mathrm{mmol} / \mathrm{L}$. At 9 weeks after $\mathrm{KT}$, the TTKG was at 3 and serum potassium level was maintained at $5.0 \mathrm{mmol} / \mathrm{L}$.

Conclusions: Identifying the uncommon causes of severe hyperkalemia may help recovery of the patient and shorten hospitalization after KT. 